\title{
Mass spectrometry and ion mobility spectrometry of G-quadruplexes. A study of solvent effects on dimer formation and structural transitions in the telomeric DNA sequence d(TAGGGTTAGGGT)
}

\author{
Ruben Ferreira, ${ }^{1}$ Adrien Marchand, ${ }^{2}$ Valérie Gabelica ${ }^{*}, 2$
}

${ }^{1}$ Department of Chemistry and Molecular Pharmacology, Institute for Research in Biomedicine (IRB Barcelona), IQAC-CSIC, CIBER-BNN, Baldiri i Reixac 10, E-08028 Barcelona (Spain).

2 Department of Chemistry, University of Liège, Allée de la Chimie Building B6C, B-4000 Liège, Belgium. *Address correspondence to: v.gabelica@ulg.ac.be

\begin{abstract}
We survey here state of the art mass spectrometry methodologies for investigating G-quadruplexes, and will illustrate them with a new study on a simple model system: the dimeric G-quadruplex of the 12-mer telomeric DNA sequence d(TAGGGTTAGGGT), which can adopt either a parallel or an antiparallel structure. We will discuss the solution conditions compatible with electrospray ionization, the quantification of complexes using ESI-MS, the interpretation of ammonium ion preservation in the complexes in the gas phase, and the use of ion mobility spectrometry to resolve ambiguities regarding the strand stoichiometry, or separate and characterize different structural isomers. We also describe that adding electrospray-compatible organic co-solvents (methanol, ethanol, isopropanol or acetonitrile) to aqueous ammonium acetate increases the stability and rate of formation of dimeric G-quadruplexes, and causes structural transitions to parallel structures. Structural changes were probed by circular dichroism and ion mobility spectrometry, and the excellent correlation between the two techniques validates the use of ion mobility to investigate G-quadruplex folding. We also demonstrate that parallel G-quadruplex structures are easier to preserve in the gas phase than antiparallel structures.
\end{abstract}




\section{Introduction}

Native electrospray ionization mass spectrometry (ESI-MS) allows unambiguous determination of the stoichiometry of supramolecular assemblies, either from synthetic or biological origin [1-3]. For biomolecules, "native" mass spectrometry requires that (1) the sample is prepared in solvents and buffers that preserve the native fold, and (2) that the mass spectrometer is tuned so as to just desolvate each complex and then preserve it until it reaches the mass analyser. The second point stems from the fact that mass spectrometry is inherently a destructive technique: the molecule is destroyed to be analysed. Nevertheless, several groups have shown, based on theory and experiments, that many structural elements of proteins and nucleic acids can be conserved in the gas phase sufficiently long to be probed before destruction (for a review, see reference [4]). Fortunately, G-quadruplexes are the nucleic acid structures that are the most prone to be preserved in the gas phase [5-7], thanks to the enhancement of hydrogen bonding (between guanines forming G-quartets) and electrostatic interactions (between the central cations and the G-quartet bases) in vacuo.

As a result, native ESI-MS has become widely used to study G-quadruplexes in solution, to determine the number of strands involved in assemblies or to detect and quantify complexes with ligands. A recent review comprehensively covers the literature on mass spectrometry of G-quadruplex DNA until 2009 [8]. Other recent reviews on the characterization of DNA-ligand interactions by mass spectrometry include extensive discussion of ligand binding to G-quadruplexes [9, 10]. In the present contribution, we decided to explain in detail the mass spectrometry-based methodologies we currently apply routinely for G-quadruplex analysis (with no ligand attached). We will also discuss in detail (1) how to quantitate G-quadruplexes using mass spectrometry, (2) how to probe structural transitions using ion mobility spectrometry, and (3) how to interpret ammonium ion preservation in the detected ions. Finally, we also discuss the first criterion of "native" mass spectrometry, namely the structure adopted in electrospray-compatible solution conditions.

All these points will be illustrated with a new mass spectrometric study of dimeric G-quadruplex formation from the 12-mer telomeric DNA sequence d(TAGGGT) $)_{2}$. In potassium solution, this sequence forms a mixture of interconverting antiparallel and parallel dimers (Figure 1) [11]. A parallel fold was also found by X-ray diffraction when this sequence was crystallized from $\mathrm{K}^{+}$solution [12]. Because ESI-MS cannot be carried out in the presence of millimolar $\mathrm{NaCl}$ or $\mathrm{KCl}$ concentrations, 
volatile ammonium salts must be used to ensure a suitable ionic strength for the nucleic acid to fold. This means that for G-quadruplex nucleic acids, these experimental conditions do not satisfy the first criterion for native mass spectrometry, because the structures adopted in $\mathrm{NH}_{4} \mathrm{OAc}$ are not necessarily the same as those adopted in $\mathrm{KCl}$. In the particular case of the 12-mer d(TAGGGT) 2 , only low amounts of dimer form in aqueous ammonium acetate [13], and a previous ion mobility study on the close analogue $\mathrm{d}(\mathrm{TTAGGG})_{2}$ showed that the dimer formed in ammonium acetate was mainly antiparallel [14].

In an effort to render the G-quadruplex structures amenable to investigation by ESI-MS more native-like, we decided to explore how the G-quadruplex structures change in ammonium solutions when electrospray-compatible co-solvents are added. There is indeed increasing evidence that molecular crowding conditions, usually simulated by the addition of co-solutes such as polyethylene glycol, favour parallel structures in the human telomeric sequence $[15,16]$. The G-quadruplex conformational transitions induced by co-solutes is generally understood as an effect of water activity $[17,18]$. Ethanol $[19,20]$ or acetonitrile $[20,21]$ were also found to favour the parallel structure in the intramolecular telomeric G-quadruplex. The present article reports dimeric G-quadruplex formation by 12-mer telomeric sequences in ammonium, in the presence of common electrospray co-solvents such as methanol, ethanol, isopropanol or acetonitrile. We found that all co-solvents increased the stability and the rate of dimeric G-quadruplex formation, and caused structural transitions towards parallel structures from ammonium acetate solutions.
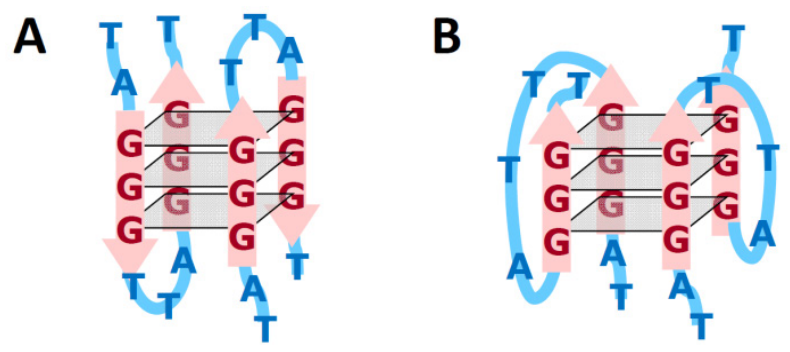

Figure 1. Schematic structures of the dimer of dTAGGGTTAGGGT in the antiparallel fold (A) and in the parallel fold (B), according to reference [11]. 


\section{Materials}

\subsection{Chemicals}

Oligodeoxynucleotides $\mathrm{dT}_{6}$ and $\mathrm{d}(\mathrm{TAGGGT})_{2}$ were purchased from Eurogentec (Belgium) and used without further purification. For all ESI-MS experiment, the sequence $\mathrm{dT}_{6}$ (monoisotopic mass 1762.318 Da) was used as an internal standard for normalizing peak intensities. Ammonium acetate (BioUltra $\sim 5 \mathrm{M}$, for molecular biology) was provided by Fluka (Sigma-Aldrich NV/SA, Bornem, Belgium), Water was nuclease-free grade from Ambion (Applied Biosystems, Lennik, Belgium). Methanol, ethanol, 2-propanol and acetonitrile were provided by Biosolve, HPLC grade.

\subsection{Circular dichroism (CD)}

CD spectra were recorded on a Jobin Yvon CD6 dichrograph using 1-cm path length quartz cells (Hellma, type No. 120-QS, France). The final concentration of oligonucleotides was $5 \mu \mathrm{M}$ in a solution containing $100 \mathrm{mM}$ ammonium acetate. For each sample, three spectra were recorded from 220 to 350 $\mathrm{nm}$ with a scan rate of $0.25 \mathrm{~nm} / \mathrm{s}$.

\subsection{Electrospray ion mobility mass spectrometry (ESI-IMS-MS)}

All mass spectrometry experiments were performed on Waters (Manchester, UK) instruments equipped with electrospray ionisation, a travelling wave ion mobility cell, and a time-of-flight mass analyser. The two instruments (Synapt G1 HDMS and Synapt G2 HDMS) were used in negative electrospray ionization and ion mobility modes. Each instrument was calibrated in the mobility mode in order to convert drift times into collision cross sections, using oligonucleotides of known collision cross sections, as described previously [22].

On the Synapt G1 HDMS, the capillary voltage was set to $-2.2 \mathrm{kV}$; cone voltage $=30 \mathrm{~V}$; extraction cone $=4 \mathrm{~V}$; source pressure $\left(\right.$ pirani reading) $=3.15$ mbar; source and desolvation temperatures $=40{ }^{\circ} \mathrm{C}$ and $60{ }^{\circ} \mathrm{C}$, respectively; trap and transfer voltages $=6 \mathrm{~V}$ and $4 \mathrm{~V}$, respectively. The ion mobility cell is filled with $\mathrm{N}_{2}$ at 0.531 mbar (pirani reading), and an electric field is applied to the cell in the form of 
waves (wave height $=8 \mathrm{~V}$ ) that pass through the cell at $300 \mathrm{~m} / \mathrm{s}$. The bias voltage for ion introduction into the IMS cell was $15 \mathrm{~V}$, unless otherwise mentioned.

On the Synapt G2 HDMS, the capillary voltage was set to $-2.2 \mathrm{kV}$; cone voltage $=30 \mathrm{~V}$; extraction cone $=4 \mathrm{~V}$; source pressure $\left(\right.$ pirani reading) $=3.25$ mbar; source and desolvation temperatures $=40{ }^{\circ} \mathrm{C}$; trap and transfer voltages $=4 \mathrm{~V}$. The helium cell is supplied with $\mathrm{He}$ at $180 \mathrm{~mL} / \mathrm{min}$, and the ion mobility cell is supplied with $\mathrm{N}_{2}$ to reach a pressure of 3.88 mbar in the IMS cell (instrument pirani reading). The wave height was $40 \mathrm{~V}$ and the wave speed was $1000 \mathrm{~m} / \mathrm{s}$. The bias voltage for ion introduction into the IMS cell was $35 \mathrm{~V}$.

The main difference between the instruments therefore lies in the ion mobility cell. The Synapt G2 HDMS has a higher resolution in ion mobility mode than the Synapt G1 HDMS. However, due to the higher nitrogen pressure and despite the presence of the helium cell at the entrance of the ion mobility cell, the ions undergo more energetic collisions prior to their entrance in the mobility cell of the Synapt G2. We will show in the results and discussion section how this can affect the preservation of the structure of the G-quadruplexes in the gas phase.

The $\mathrm{d}(\mathrm{TAGGGT})_{2}$ stock was single strand concentration of $200 \mu \mathrm{M}$ in water and annealed by heating to $85^{\circ} \mathrm{C}$ and slowly cooling to room temperature before use. To follow the kinetics of dimer formation, the samples were prepared at room temperature $\left(22 \pm 1{ }^{\circ} \mathrm{C}\right)$ and injected at a final single strand concentration of $5 \mu \mathrm{M} \mathrm{dT}_{6}$ and $5 \mu \mathrm{M} \mathrm{d}\left(\mathrm{TAGGGT}_{2}\right.$ at a rate of $140 \mu \mathrm{L} / \mathrm{h}$. The kinetics of dimerization was tested in $20 \%, 40 \%, 60 \%$ and $80 \%$ volume percentage of co-solvent (methanol, ethanol, 2-propanol and acetonitrile), the rest of the solvent being aqueous ammonium acetate (100 $\mathrm{mM}$ ). Adequate volumes of aqueous single strand, water, and co-solvents were pre-mixed and allowed to equilibrate $10 \mathrm{~min}$ at room temperature, and ammonium acetate (from a $1 \mathrm{M}$ stock solution) was added last, to initiate G-quadruplex formation. The mass spectral recording was started simultaneously with ammonium addition. The sample was thoroughly mixed and loaded into the 250 -mL syringe, the spray was initiated as quickly as possible by manually pushing the syringe, and the flow rate was then stabilized at $140 \mu \mathrm{L} / \mathrm{h}$. The time lapse between ammonium addition and spray stabilization is typically $1 \mathrm{~min}$. The dimer formation can also be triggered either adding the co-solvent, but this is less adequate for accurate kinetics analysis, because the solution temperature transiently changes due to the endothermicity (in the case of $\mathrm{ACN}$ ) or exothermicity (in the case of alcohols) of solvent mixing. All 
time-resolved experiments reported here for the determination of the response factors (see section 4) were therefore triggered by ammonium acetate addition, and performed on the Synapt G1 HDMS spectrometer.

\section{Effect of electrospray-compatible organic co-solvents on G-quadruplex assembly in ammonium acetate solution}

ESI-MS of nucleic acids from purely aqueous ammonium acetate in the negative mode often gives low ion signals, as compared for example to ESI-MS of proteins in the positive ion mode. This is probably one of the reasons why ESI-MS of nucleic acid complexes is much less widespread than ESIMS of protein complexes. A typical trick to enhance ion response in ESI-MS is to add to the sample some organic co-solvents more volatile than water in order to aid droplet desolvation and increase the signal-to-noise ratio. Since the early days [23], we took the habit to add $20 \%$ or $10 \%$ methanol to analyse nucleic acid complexes by ESI-MS, and most mass spectrometrists adopt similar recipes, using for example $10 \%$ isopropanol $[24,25]$ or 20-25\% methanol [26-30]. Some papers even report fair MS spectra of G-quadruplexes higher-order assembly and ligand binding using up to 50\% methanol [3133]. In contrast, published G-quadruplex MS spectra recorded in purely aqueous ammonium acetate solutions [34-36] often show lower signal-to-noise ratio. Porter and Beck recently mentioned that slight solvent-induced changes in G-quadruplexes were evidenced by ion mobility spectrometry, but the solvent effect was not extensively studied [37]. It is therefore very tempting to systematically add low amounts of organic co-solvents to perform ESI-MS of nucleic acid complexes, and in our past methodological reviews we were also recommending adding 10-20\% methanol "just prior to ESI-MS analysis" $[10,38]$.

However, in a recent ESI-MS study of the self-assembly of the tetramolecular $\left[\mathrm{dTG}_{5} \mathrm{~T}\right]_{4}$ Gquadruplex, we observed that the methanol content had a dramatic influence on the rate of Gquadruplex formation: the higher the methanol percentage, the faster the G-quadruplex assembly [39]. This prompted us to systematically check before publishing results obtained in $20 \%$ methanol that similar results were also obtained with 100\% aqueous solution. Most often the results agree and the spectra obtained with organic co-solvent show only high signal-to-noise ratio, but sometimes the results are not equivalent, as strikingly demonstrated below. 
Here we studied the effect of common electrospray co-solvents like methanol $(\mathrm{MeOH})$, ethanol $(\mathrm{EtOH})$, isopropanol $(\mathrm{iPrOH})$ or acetonitrile $(\mathrm{ACN})$ on the dimeric G-quadruplex formation by a 12mer. At this point, we cannot conclude that we have a G-quadruplex structure from the sole fact that we detect a dimer. Further evidence that we are indeed in the presence of G-quadruplex structures come from ion mobility spectrometry experiments (see section 5), from the ammonium ion preservation (see section 6) and from correlations with other solution-phase methods. The telomeric sequence $\mathrm{d}(\mathrm{TAGGGT})_{2}$ does not form significant amounts of dimer when annealed at $5 \mu \mathrm{M}$ strand concentration in purely aqueous $100 \mathrm{mM} \mathrm{NH}{ }_{4} \mathrm{OAc}$. A dimer forms however when co-solvents are added to this solution, as revealed by the electrospray mass spectra (Figure 2). The amount of dimer increases with the percentage of organic co-solvent (see Fig. 2 from A to D for EtOH). The spectrum obtained in purely aqueous solution is shown in Figure 1E for comparison. The other co-solvents, at $60 \%$ volume percentage (Fig. 2F for MeOH, Fig. 2G for iPrOH, Fig. 2H for ACN), also favour the dimer formation. In all cases, the mass-to-charge ratio of the peaks corresponding to the dimer indicate the preferential preservation of two ammonium ions, presumably the two ions trapped in between the three G-quartets. Ammonium ion preservation will be further discussed in section 6 .

Very similar monomer/dimer spectral intensity ratios were obtained after days of reaction or immediately after annealing, showing that the effect of co-solvent is not only a kinetic effect, but also a thermodynamic effect. Structural transitions are also observed within the population of dimer, depending on the solvent and on the reaction time, as will be discussed in detail in section 5 . To conclude the present section, we emphasize that the addition of co-solvents to the electrospray samples prior to analysis should be given greater attention than in the past, now that several studies in solution documented that co-solvents can dramatically affect G-quadruplex structure and self-assembly state [17-21, 39]. Addition of co-solvents, actually dehydration [17, 18], favours G-quadruplex structures. Therefore, the co-solvents not only increase all ESI-MS signals thanks to better droplet desolvation, but they also increase the G-quadruplex signals simply because more are formed in solution. 


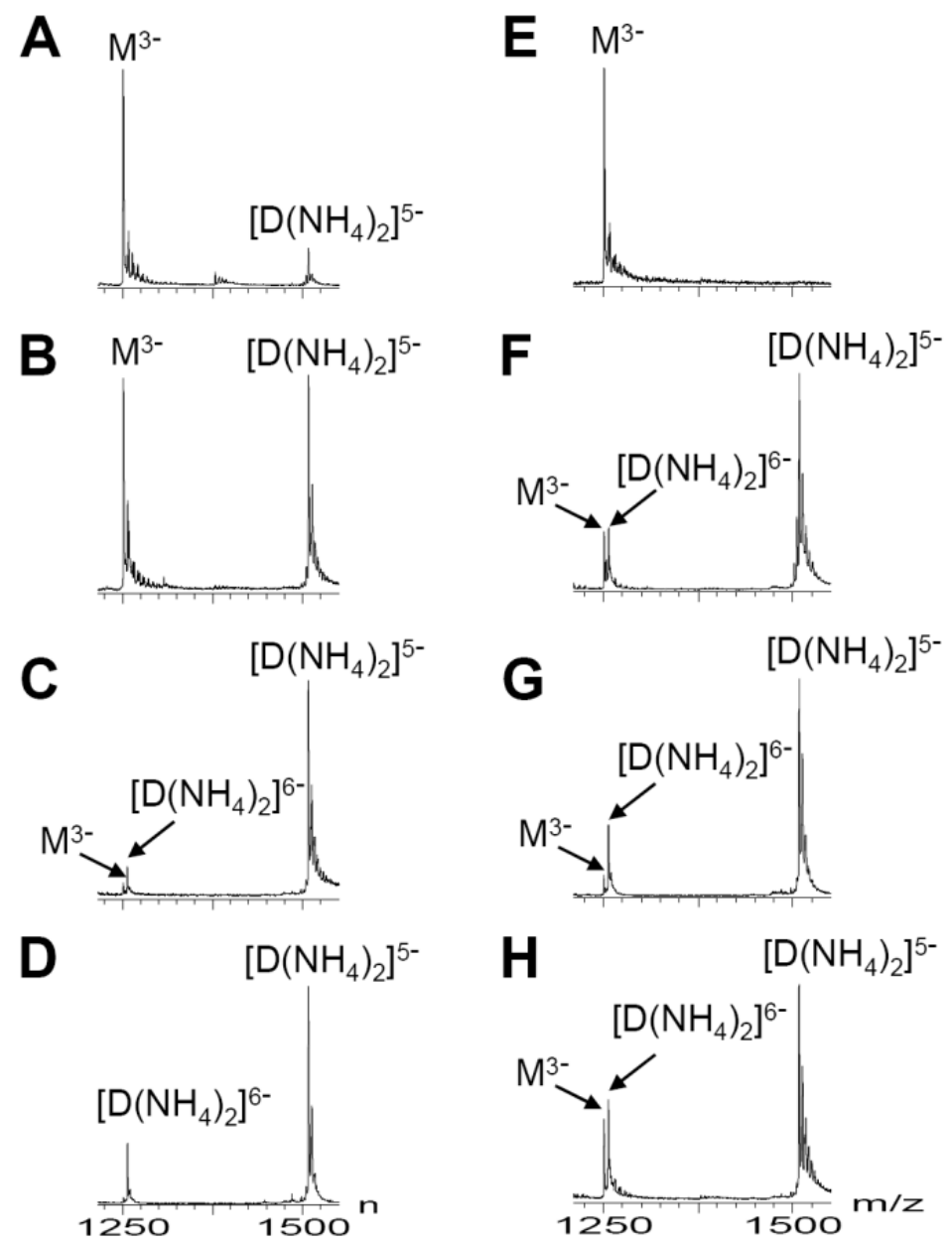

Figure 2. Electrospray mass spectra of $5 \mu \mathrm{M}$ telomeric sequence dTAGGGTTAGGGT recorded 1 day at room temperature after preparation in (A) 20/80 (v:v) EtOH/aqueous $\mathrm{NH}_{4} \mathrm{OAc} 100 \mathrm{mM}$, (B) 40/60 (v:v)

EtOH/aqueous $\mathrm{NH}_{4} \mathrm{OAc} 100 \mathrm{mM}$ (C) 60/40 (v:v) EtOH/aqueous $\mathrm{NH}_{4} \mathrm{OAc} 100$ mM, (D) 80/20 (v:v) EtOH/100 mM aqueous $\mathrm{NH}_{4} \mathrm{OAc}$, (E) 100\% aqueous $\mathrm{NH}_{4} \mathrm{OAc} 100$ mM, (F) 60/40 (v:v) MeOH/aqueous $\mathrm{NH}_{4} \mathrm{OAc} 100$ mM, (G) 60/40 (v:v) iPrOH/aqueous $\mathrm{NH}_{4} \mathrm{OAc} 100$ mM, (H) 60/40 (v:v) ACN/aqueous $\mathrm{NH}_{4} \mathrm{OAc} 100 \mathrm{mM}$. M stands for the monomer, D stands for the dimer, which is observed predominantly with two ammonium ions preserved. 


\section{Quantitative mass spectrometry: determination of absolute concentrations of monomer and dimer from relative peak intensities}

The factor relating the peak intensity of a compound to its concentration is called the response factor [40-46]. In order to determine the absolute concentrations of monomer and dimer from their relative intensities, we therefore need to determine the relative response of the monomer and the dimer. To this aim, we use the internal standard method described in more detail elsewhere [46]. For all nucleic acid response factor determinations, we use a short polythymine oligonucleotide, here $\mathrm{dT}_{6}$ at 5 $\mu \mathrm{M}$ concentration, as internal standard. To determine the monomer and dimer relative response with respect to this internal standard, we need a range of conditions where their relative abundances vary and where the total strand concentration (and therefore the mass balance equation) is known. The ideal situation is therefore to follow the dimer and monomer signals with respect to the internal standard in a kinetics experiment: the sample is identical in the whole experiments, except that the monomer is the most abundant at the beginning and the dimer is most abundant at the end of the recording.

In $100 \mathrm{mM}$ ammonium acetate, the 12-mer sequence $\mathrm{d}(\mathrm{TAGGGT})_{2}$ is mainly present as a monomer. The dimer formation can be triggered either by adding the co-solvent, or by adding the ammonium acetate. Both types of experiments were performed, and the relative intensity of dimer formed at the end point was the same, but transient temperature variations of the solution upon waterco-solvent mixing are detrimental to accurate kinetics studies. All kinetics experiments shown here and used to extract rate constants were therefore triggered by ammonium acetate addition. This procedure should be preferred to avoid large temperature changes of the solution shortly after solvent mixing, because the temperature of the solution might affect electrospray response. The relative response factors of the dimer compared with the single strand were determined following a previously described procedure, except that here the additional ion mobility separation further helps to extract the signals of individual species. This step is therefore described in detail below.

The peak areas of each species was extracted as a function of the "retention time" (here the reaction time) using Driftscope 2.0, as illustrated in Figure 3. The 2D-graph in panel A represents the ion abundance (darkness) as a function of the mass-to-charge $(\mathrm{m} / \mathrm{z})$ ratio on the $\mathrm{X}$-axis and the drift time in the ion mobility cell on the $\mathrm{y}$-axis. The projection on the $\mathrm{x}$-axis is the mass spectrum (top of panel A). 
Driftscope software allows to extract ion signal of a portion of the $2 \mathrm{D}$ plot as a function of the "retention time" (here, equal to the reaction time). For example, panel B shows the signal of the internal standard, which is constant over the reaction time. Ion mobility separation is particularly useful to distinguish species that overlap in mass/charge ratio, such as the $\left[\right.$ monomer] ${ }^{3-}$ from the $[\text { dimer }]^{6-}$ (rectangles $\mathrm{C}$ and $\mathrm{D}$, respectively) and allows to extract the different signals, even if one of the species was less abundant. The total dimer and monomer signals as a function of reaction time are obtained by additioning all their respective populations.

The response factors were then determined as described previously [46], by solving the matrix expressing the mass balance equation $([\mathrm{M}]+2[\mathrm{D}]=5 \mu \mathrm{M})$ at each time point. We determined the ratio between the response of the dimer (sum of peak areas of charge states 6-, 5- and 4-) and the response the single strand (sum of peak areas of charge states 4- and 3-), for the sequence d(TAGGGT) 2 discussed in detail here, as well as for the derivative sequences d(TTAGGG) $)_{2}$ d(AGGGTT) 2 and $\mathrm{d}(\text { GGATTT) })_{2}$ (not shown). We found that the dimer/monomer response ratio was equal to $1.3 \pm 0.4$ for all sequences, co-solvents, and relative volume percentages. Consequently, the relative response is not very sensitive to the antiparallel/parallel structure (see below) of the G-quadruplex. It also demonstrates that the increased sensitivity when a co-solvent is added to water is due to an increase of signal for both the monomer and dimer, by a similar factor. The response factors above were calculated for the peak areas of all charge states. We therefore highlight again that, although we find responses of similar order of magnitude for the monomer and the dimer, relative peak heights of the most intense charge states (readily evaluated at the naked eye in the mass spectra) do not necessarily reflect relative abundances in solution. The concentrations of monomer and dimer, respectively, were recalculated using the response factors and are shown in Figure 3E. 


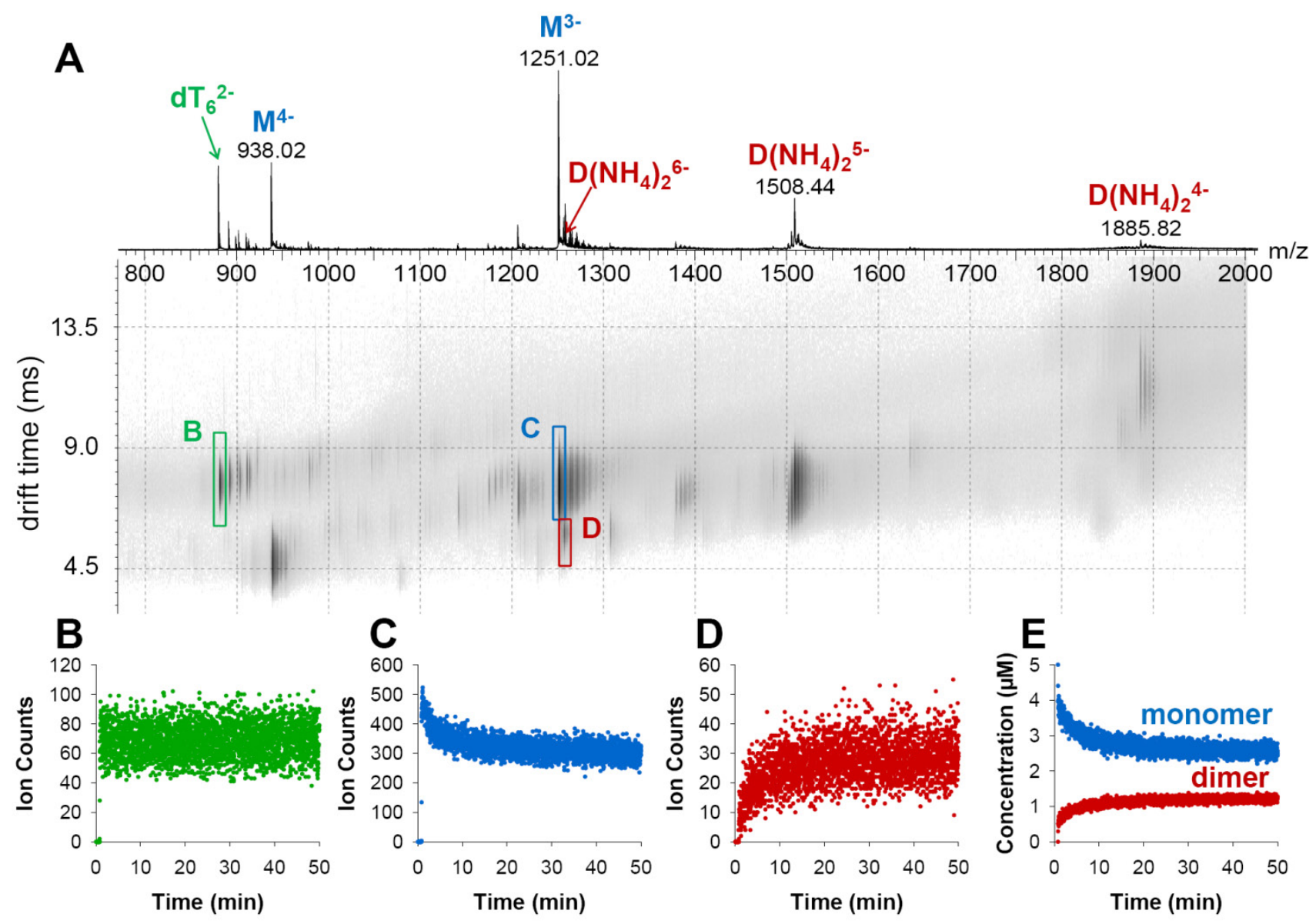

Figure 3. Illustration of the utility of drift time separation in the ion mobility cell to distinguish single-stranded monomer (M) and G-quadruplex dimer $\left(\mathrm{D}\left(\mathrm{NH}_{4}\right)_{2}\right)$ and extract their respective peak areas. (A) 2D graph showing the total ion abundance (darkness) as a function of the $\mathrm{m} / \mathrm{z}$ and of the drift time, obtained in the kinetics experiment, from 0 to $50 \mathrm{~min}$, of $5 \mu \mathrm{M}$ d(TAGGGT) ${ }_{2}$ folding in 40/60 (v:v) EtOH/100 $\mathrm{mM}$ aqueous ammonium acetate. Note that the mass spectral intensities differ from those of Figure 1B because the latter were acquired after 1 day of folding. Panels (B), (C) and (D) show the extracted ion signals as a function of the reaction times

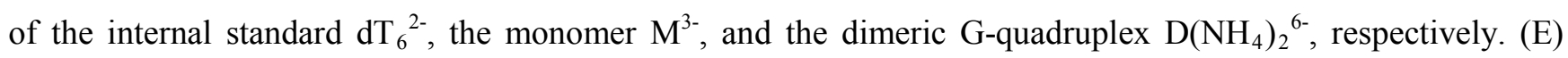
Time evolution of the concentrations of monomer and dimer, recalculated using the average relative response factor found in $\mathrm{EtOH}\left(\mathrm{R}_{\operatorname{monomer}(3-\text { and 4-) }} / \mathrm{R}_{\text {dimer(4- to 6-) }}=1.25\right)$. 


\section{Parallel G-quadruplex structures are preserved in the gas phase: comparison between circular dichroism spectroscopy and ion mobility spectrometry}

The CD spectrum provides information about the strand orientation (parallel, antiparallel, or hybrid) of G-quadruplexes, because the stacking of consecutive G-quartets is related to the strand orientation and to the syn/anti orientation about glycosilic bonds [47]. Purely parallel-stranded structures exhibit a positive CD peak around $260 \mathrm{~nm}$ and a negative peak around $240 \mathrm{~nm}$, whereas purely antiparallel-stranded structures exhibit a positive peak around $295 \mathrm{~nm}$ and a negative peak around $260 \mathrm{~nm}$. Figure 4A and 4B show the circular dichroism spectra of the structures formed by the sequence d(TAGGGT) $)_{2}$ after 5 minutes, 1 hour, or 1 day at room temperature in ethanol (4A) or methanol (4B). Clearly, a mixture of parallel and antiparallel G-quadruplexes is formed first, and the mixture slowly converts to a parallel structure at longer times. Also, the conversion to a parallel structure in solution is much faster in ethanol than in methanol. Results obtained in acetonitrile and isopropanol (not shown) resemble those obtained in ethanol.

The different folding of this sequence in different solvents constitutes an ideal case to validate whether ion mobility spectrometry can be used to obtain structural information. Mass spectrometry and ion mobility spectrometry should ideally provide snapshots of the solution-phase conformations. The condition is that each structure is preserved in the gas phase. The ion mobility cell separates ions according to their mobility, i.e. the ratio between their velocity in a bath gas and the electric field causing that movement. The ion mobility depends on ion properties such as its charge (the mobility increases, and thereby the drift time decreases when the charge $z$ increases) and its collision cross section (the mobility decreases, and thereby the drift time increases when the collision cross section noted CCS or $\Omega$ - increases). The collision cross section is the orientationally averaged surface of the ion that is exposed to collisions with the bath gas, and is expressed in $\AA^{2}$. Antiparallel and parallel structures can therefore be differentiated by ion mobility spectrometry provided that (1) these structures have significantly different collision cross sections and that (2) the structures formed in solution are preserved by the multiply charged anions in the gas phase on the time scale of the experiment (several milliseconds).

A previous publication has predicted by theoretical calculations that the dimer of $\mathrm{d}(\mathrm{TTAGGG})_{2}$ would have a CCS of $785 \AA^{2}$ in its antiparallel form, and a CCS of $845 \AA^{2}$ in its parallel form [14]. Our 
dimer of d(TAGGGT) $)_{2}$ has the same base composition, and similar CCS values are anticipated. The distribution of CCS were determined for the dimer [d(TAGGGT) $\left.]_{2}\right]_{2}{ }^{5-}$ after $5 \mathrm{~min}, 1$ hour and 1 day, with two different instruments: the Synapt G1 HDMS (Figure 4C: ethanol; Figure 4D: methanol) and the Synapt G2 HDMS (Figure 4E: ethanol; Figure 4F: methanol). On both instruments, we see that the contribution corresponding to a parallel G-quadruplex dimer in the gas phase $\left(845 \AA^{2}\right)$ increases when the abundance of the parallel structure in solution, as indicated by the CD spectra, increases. However, the contribution of the parallel G-quadruplex is much more clearly seen with the Synapt G2 instruments, thanks to its higher mobility resolution.
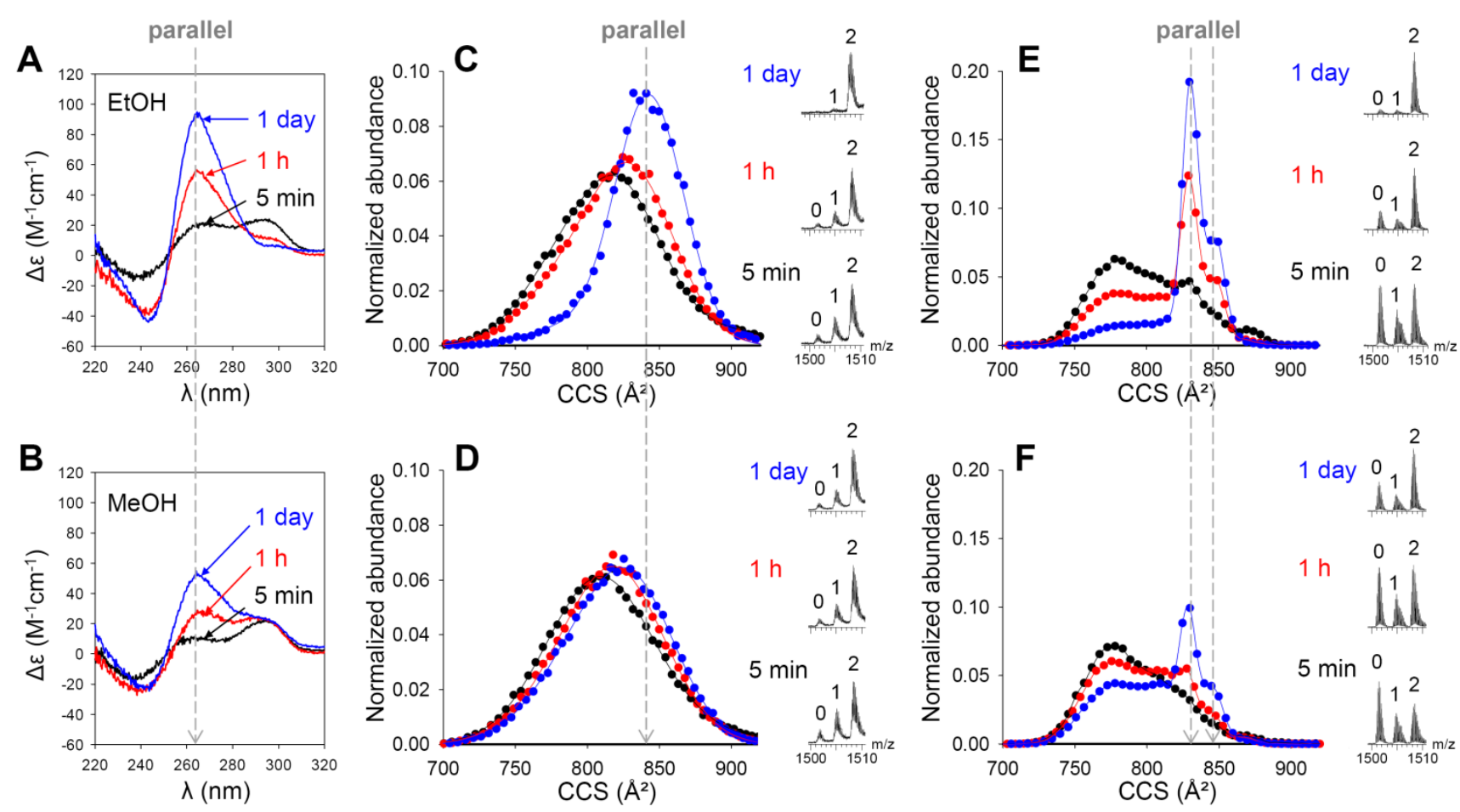

Figure 4. Comparison between the dimers formed in ethanol and methanol, as a function of folding time. (A-B) Circular dichroism spectra in (A) EtOH and (B) $\mathrm{MeOH}$ recorded 5 min (black), $1 \mathrm{~h}$ (red) and $24 \mathrm{~h}$ (pink) after preparation in $60 / 40(\mathrm{v}: \mathrm{v})$ co-solvent/aqueous $\mathrm{NH}_{4} \mathrm{OAc} 100 \mathrm{mM}$. The arrows indicate the peaks attributable to parallel (para) and antiparallel (anti) strand arrangement, respectively. (C-D) Collision cross section population obtained with the Synapt G1 HDMS instrument for the [Dimer] $]^{5-}$ (total from 0 to 2 ammonium ions preserved) sprayed from the same sample solutions; (C) EtOH; (D) MeOH. The CCS distributions were normalized by their total area. The insets show the distribution of number of preserved ammonium ions in the corresponding mass spectra. (E-F) Same as C-D but obtained with the higher-resolution Synapt G2 HDMS instrument. 
In summary, the mass spectra show that a dimer can form both in ethanol and in methanol (Figures $2 \mathrm{C}$ and $2 \mathrm{~F}$ in $60 \%$ ethanol and methanol, respectively). The ion mobility spectra (Figure 4 ) reveal that the structure(s) formed in methanol tend to be less parallel than in ethanol, and these interpretations have been validated by circular dichroism experiments. The dimer structure also changes with the reaction time. In all solvents, antiparallel or mixed structures form first. The population then slowly shifts to a parallel fold, and this conversion is slower in methanol than in the other solvents. Refining the interpretation of the ion mobility peak positions and widths would however require additional modelling on the different structures potentially formed by each sequence, and is beyond the scope of the present paper. For example, two ion mobility peaks $\left(830 \AA^{2}\right.$ and $\left.847 \AA^{2}\right)$ can be distinguished for $\left[\mathrm{d}(\mathrm{TAGGGT})_{2}\right]_{2}{ }^{5-}$ dimer with the high-resolution instrument, and both arise when the population of parallel structure increases. Molecular modelling would allow proposal of structures compatible to each of these average collision cross sections, but we anticipate that further developments are needed first on the parameterization of collision cross section calculations for nucleic acids.

\section{Inner ammonium ions preservation is correlated with the structure: antiparallel structures are more labile in the gas phase than parallel structures}

From the ion mobility results, we concluded that the parallel structure was preserved when transferred from the solution to the gas phase, but the results for the antiparallel structure were less clear. The reason is that, in addition to a modification of the collision cross section distribution depending on the solution structures, we also see a modification of the distribution of number of preserved ammonium ions depending on the solution conditions. The insets of panels $4 \mathrm{C}$ to $4 \mathrm{~F}$ show the ammonium ion distributions from which the CCS distributions were reconstructed. When the predominant structure in solution is a parallel quadruplex, the ammonium ion distribution becomes more biased towards two ammonium ions. Moreover, quite strikingly, the ammonium ion number distribution varies from instrument to instrument (compare 4C with 4E, and 4D with 4F). In this section, we will first discuss, for those not familiar with mass spectrometry instrumentation, what makes the mass spectra appear so different although the samples are the same. Then we will discuss how ammonium ion distributions and collision cross section distributions can be interpreted in terms of structure. 
Why do (G-quadruplex) mass spectra look different (in terms of ammonium ion preservation) when recorded on different instruments? The answer to this very general question (read the previous sentence without the parentheses) is that ions can acquire different amounts of internal energy [48] for different amounts of time, depending on the collisions they undergo in the instrument. Inelastic collisions indeed redistribute part of the relative translation energy into vibrational energy of the ion [49]. The relative translation energy depends on ion speed before the collision, and therefore increases when a potential (voltage) difference is increased in a region of the instrument where collisions can occur. Therefore, the distribution of internal energy acquired by the ion population depends both on the hardware configuration of the instrument (pumping system, shape of metal pieces between which voltage differences are applied), and on the experimental parameters (values of voltages and pressures, nature of the collision gas) [50]. When the internal energy distribution of an ion population increases, the internal energy increases, and in other words the ions get more vibrational energy and start exploring more conformations on their energy landscape, starting with free rotations at low internal energy and more and more energy-costly changes as the energy increases. Ultimately, this can lead to conformational changes, chemical reactions such as proton transfer, and irreversible dissociation [4]. The consequence is that, because of internal energy differences, not only mass spectra, but also ion mobility distributions can look different when recorded on different instruments.

A change in the ion mobility distribution when the internal energy is increased indicates an isomerization in the gas phase involving conformations of sufficiently different collision cross sections. "Ammonium ion" loss when the internal energy is increased actually indicates a proton transfer from the ammonium ion to the DNA strand followed by the irreversible loss of $\mathrm{NH}_{3}$. Both are consequences of internal energy increase upon voltage increase. Let us now examine whether ammonium ion loss and conformational changes are linked. The dimer $\left[\mathrm{d}(\mathrm{TAGGGT})_{2}\right]_{2}$ provides an excellent model system, because depending on the solvent, either a purely parallel structure (in ethanol) or a mixture of parallel and antiparallel structures (in methanol) can be formed in solution after 1 day. Figure 5 shows the 2D mass/mobility plots of the [dimer] ${ }^{5-}$ sprayed from methanol (Figure 5A-D) or from ethanol (Figure 5E$\mathrm{DH}$ ), when the IMS bias voltage of the Synapt G1 HDMS instrument is increased. The bias is the voltage difference accelerating the ions towards the ion mobility cell. A non-zero voltage is needed for the ions to enter the mobility cell, which is at higher pressure than the zone upstream. However, the higher the bias voltage, the more energetic the collisions occurring just before the entrance in the mobility cell. 


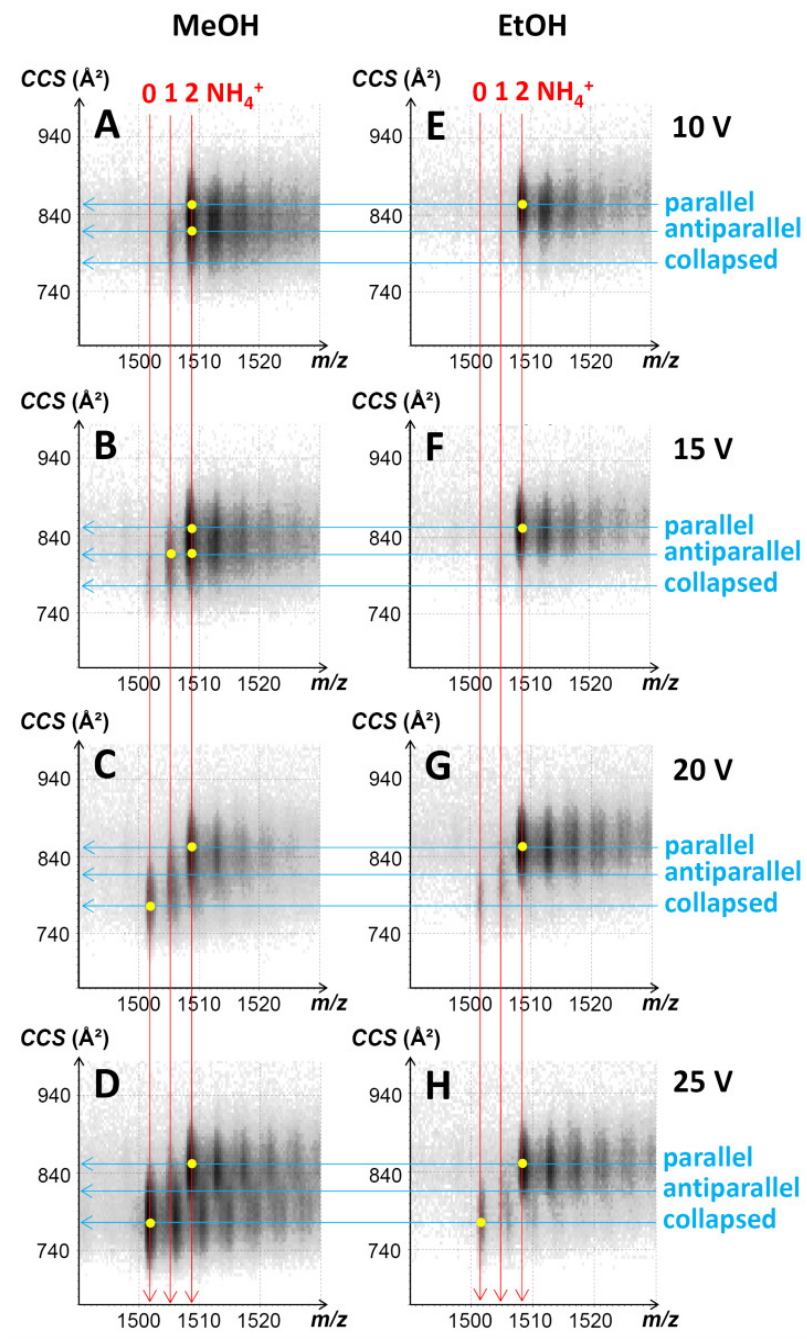

Figure 5. Influence of the bias voltage of the Synapt G1 HDMS instrument (from $10 \mathrm{~V}$ at the top to $25 \mathrm{~V}$ at the bottom) on the ammonium ion distribution and on the collision cross section of the dimers formed (A-D) after 1 day in $60 \%$ methanol or (E-H) after 1 day in $60 \%$ ethanol. The guidelines in red indicate the masses of the dimer with 2, 1 or zero ammonium ions preserved, and the guidelines in blue indicate the interpretation of collision cross sections in terms of dimer structure (see main text).

The bidimensional separation allows correlation of ammonium ion loss (differentiated based on the $m / z$; $\mathrm{x}$-axis) upon internal energy increase with conformational changes, indicated by changes in the collision cross section (CCS; y-axis). At low voltage (low internal energy, Fig. 5A and 5E), the ion structures are the least likely to have been disturbed. The two ammonium ions are indeed mostly preserved (both from the methanol and the ethanol sample), and the collision cross section distributions indicate a purely parallel structure preserved in ethanol (Fig. 5E) and a mixture of parallel and antiparallel from methanol (Fig. 5A). The latter interpretation is validated by both the fairly good 
agreement with calculated collision cross sections of parallel and antiparallel structures (845 and 785 $\AA^{2}$, respectively), and the circular dichroism data of the respective starting solutions (blue spectra in Fig. 4A and 4B for ethanol and methanol, respectively). When the internal energy is increased, ammonium loss is observed mostly in the case of the methanol sample (bias voltage increased from Fig. 5A to 5D), and much less in the case of the ethanol sample (bias voltage increased from Fig. 5E to $5 \mathrm{H})$. The collision cross section analysis of each mass spectral peak reveals that, independently of the starting solvent, the parallel structure is preserved in the gas phase at high voltage with two ammonium ions. In the methanol sample, the fraction that has lost ammonium ions at moderate voltage had come mostly from the antiparallel structure. At $15 \mathrm{~V}$ (Fig. 5B), an intermediate is seen, with 1 ammonium ion and a presumably antiparallel structure. In all cases, the species with zero ammonium ions have always an even smaller collision cross section, presumably indicating a collapse into a globular structure following the ammonia loss.

Now that the influence of the internal energy on the ammonium ion preservation and structure preservation has been explained in detail, we can understand the origin of the differences obtained between the Synapt G1 HDMS instrument (Figure 4C-D, with a bias voltage = 15V) and the Synapt G2 HDMS instrument (Figure 4C-D, with a bias voltage $=35 \mathrm{~V}$ ). The Synapt G2 instrument imparts more internal energy to the ions that the Synapt G1. This is due to the higher pressure in the IMS cell that requires using higher bias voltages to ensure ion transfer. The bias voltage of $35 \mathrm{~V}$ is the minimum value to obtain decently intense ion signals. Figure 6 shows the 2D plots obtained with the Synapt G2, to be compared with those obtained with the Synapt G1 on the same samples (Figure 5). The minimum internal energy imparted to the [dimer $]^{5-}$ in the Synapt G2 (Figure 6) is equivalent to that imparted at approximately $25 \mathrm{~V}$ on the Synapt G1 (Figures 5D and 5H). Hence, the higher mobility resolution attainable in the Synapt G2 HDMS comes at the price of more internal energy imparted to the ions before the analysis, which might disrupt the most fragile structures (here the antiparallel structure). This highlights the importance of instrument choice and of carrying out voltage-dependent experiments to grasp internal energy effects on the mass spectra and the ion mobility spectra. 


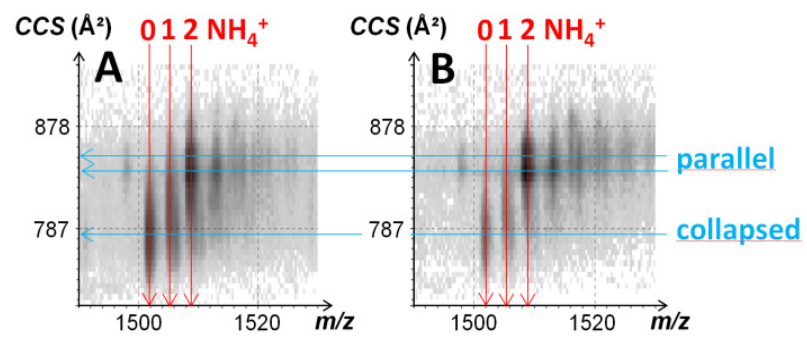

Figure 6. 2D plot of ammonium ion distribution and collision cross section of the dimers observed on the Synapt G2 HDMS spectrometer (bias $=35 \mathrm{~V}$ ), formed after 1 day in (A) $60 \%$ methanol or (B) $60 \%$ ethanol.

In summary:

(1) Only the structures with ammonium ions preserved between their G-quartets are likely to have structural elements preserved from the initial solution.

(2) Ammonia loss is closely linked to, and most likely just precedes the loss of G-quadruplex structure upon internal energy increase. Importantly, because ammonia loss is irreversible, no interconversion from one G-quadruplex structure to another G-quadruplex structure can occur in the gas phase. However, both structures can be disrupted upon ammonia loss.

(3) Parallel structures in the gas phase are more resistant to ammonia loss than antiparallel structures. Although this was suggested in previous works by comparison between different sequences [13], this is now shown unambiguously with two structures formed from the same sequence. Ammonia loss requires proton transfer to the DNA. Therefore, the probability of ammonia loss depends on the accessibility proton exchange partners close to the terminal G-quartets. Therefore, faster ammonia loss for antiparallel structures may be due to the presence of more accessible lateral or diagonal loops, and/or to an intrinsically higher degree of conformational fluctuations of antiparallel structures compared to parallel structures in the gas phase.

(4) Ammonium ion preservation depends not only on the structure, but also on the instrument and on experimental parameters such as voltages. Therefore, before concluding on G-quadruplex structure based solely on the preservation of inner ammonium ions, a voltage-dependent analysis must be performed, and ideally ion mobility spectrometry should be used to interpret the results. Conversely, moderately activating conditions can be exploited to intentionally disrupt some structures while preserving others. For example, the ammonium ion distribution obtained with the Synapt G2 instrumental conditions (Figure 4E-F) actually easily allow to discriminate the parallel dimer ( 2 ammonium ions preserved) from the antiparallel one ( 0 or 1 ammonium ions). 


\section{Conclusions and outlook}

In conclusion, the results above demonstrate the strong correlation between ammonium ion preservation and structure preservation in the gas phase. Parallel G-quadruplex structures are generally found to be more stable in the gas phase than antiparallel structures, both in terms of ammonium ion preservation and tridimensional structure preservation as measured by ion mobility spectrometry. However, antiparallel structures are more labile in the gas phase, and one cannot straightforwardly conclude from the absence of preserved ammonium in the gas phase that no G-quadruplex structure was present in solution. Future work will specifically address the question of antiparallel structure preservation.

The last two sections of the manuscript explained how to interpret bidimensional mass spectrometry/ion mobility spectrometry experiments in terms of structure. The present mixture was fairly simple (one strand forming a monomer and a dimer, plus another strand as internal standard), and only a single peak, the [dimer $]^{5-}$ of the DNA sequence d(TAGGGT) ${ }_{2}$, was analysed in detail. Similar analysis can in principle be carried out for each peak resolvable in a mass spectrum. The power of mass spectrometry compared to other spectroscopic techniques in solution clearly lies in the possibility of carrying out such structural studies on each species present in a complex mixture, and we hope we have provided some guidelines for mass spectrometry and ion mobility data interpretation.

We have shown that the addition of co-solvents in ammonium acetate can influence G-quadruplex formation and structural transitions. The main result is that electrospray-compatible organic co-solvents can favour the formation of G-quadruplexes, and that the structure obtained depends both on the nature of the solvent and on the reaction time. The temporal evolution of the abundance of dimer of each structure has been studied in detail by ESI-IMS-MS for different sequences and solvents, and the results of this thermodynamic and kinetic analysis as a function of the water activity will be published elsewhere. Organic co-solvent addition is an easy way to generate different folds in solution and make them amenable to mass spectrometry and ion mobility analysis. Future work will also be devoted to study the ligand binding preference for antiparallel versus parallel structures, using ESI-IMS-MS. 


\section{Acknowledgements}

This work was supported by the Fonds de la Recherche Scientifique-FNRS (research associate position, and FRFC grant 2.4528.11), the EU COST action (MP0802, STSM 9145 to RF), and the Spanish Ministry of Science (CTQ-2010-20541). The authors acknowledge the GIGA-Proteomics platform for access to the instruments, Hisae Tateishi-Karimata and Daisuke Miyoshi for useful comments on a preliminary version of this manuscript, and Ramon Eritja for proofreading the manuscript.

\section{References}

[1] J.A.Loo. Studying non-covalent protein complexes by electrospray ionization mass spectrometry, Mass Spectrom. Rev. 16 (1997) 1-23.

[2] R.D.Smith, J.E.Bruce, Q.Wu, Q.P.Lei. New mass spectrometric methods for the study of noncovalent associations of biopolymers, Chem. Soc. Rev. 26 (1997) 191-202.

[3] A.J.R.Heck, R.H.H.Van Den Heuvel. Investigation of intact protein complexes by mass spectrometry, Mass Spectrom. Rev. 23 (2004) 368-389.

[4] K.Breuker, F.W.McLafferty. Stepwise evolution of protein native structure with electrospray into the gas phase, $10^{-12}$ to $10^{2} \mathrm{~s}$, Proc. Natl. Acad. Sci. U. S A 105 (2008) 18145-18152.

[5] M.Rueda, F.J.Luque, M.Orozco. G-quadruplexes can maintain their structure in the gas phase, J. Am. Chem. Soc. 128 (2006) 3608-3619.

[6] J.Sponer, N.Spackova. Molecular dynamics simulations and their application to four-stranded DNA, Methods 43 (2007) 278-290.

[7] E.Fadrna, N.Spackova, R.Stefl, J.Koca, T.E.Cheatham, III, J.Sponer. Molecular dynamics simulations of guanine quadruplex loops: advances and force field limitations, Biophys. J. 87 (2004) 227-242.

[8] G.Yuan, Q.Zhang, J.Zhou, H.Li. Mass spectrometry of G-quadruplex DNA: Formation, recognition, property, conversion, and conformation, Mass Spectrom. Rev. 30 (2011) 11211142 .

[9] J.S.Brodbelt. Evaluation of DNA/Ligand interactions by electrospray ionization mass spectrometry, Annu. Rev. Anal. Chem. 3 (2010) 67-87.

[10] F.Rosu, E.De Pauw, V.Gabelica. Electrospray mass spectrometry to study drug-nucleic acid interactions, Biochimie 90 (2008) 1074-1087. 
[11] A.-T.Phan, D.J.Patel. Two-repeat human telomeric d(TAGGGTTAGGGT) sequence forms interconverting parallel and antiparallel G-quadruplexes in solution: Distinct topologies, thermodynamic properties, and folding/unfolding kinetics, J. Am. Chem. Soc. 125 (2003) 15021-15027.

[12] G.N.Parkinson, M.P.H.Lee, S.Neidle. Crystal structure of parallel quadruplexes from human telomeric DNA, Nature 417 (2002) 876-880.

[13] G.W.Collie, G.N.Parkinson, S.Neidle, F.Rosu, E.De Pauw, V.Gabelica. Electrospray Mass Spectrometry of Telomeric RNA (TERRA) Reveals the Formation of Stable Multimeric GQuadruplex Structures, J. Am. Chem. Soc. (2010) doi: 10.1021/ja100345z.

[14] E.S.Baker, S.L.Bernstein, V.Gabelica, E.De Pauw, M.T.Bowers. G-quadruplexes in telomeric repeats are conserved in a solvent-free environment, Int. J. Mass Spectrom. 253 (2006) 225237.

[15] D.Miyoshi, H.Karimata, N.Sugimoto. Drastic effect of a single base difference between human and Tetrahymena telomere sequences on their structures under molecular crowding conditions, Angew. Chem. Int. Ed. 44 (2005) 3740-3744.

[16] Y.Xue, Z.Y.Kan, Q.Wang, Y.Yao, J.Liu, Y.H.Hao, Z.Tan. Human telomeric DNA forms parallelstranded intramolecular G-quadruplex in $\mathrm{K}^{+}$solution under molecular crowding condition, $\mathrm{J}$. Am. Chem. Soc. 129 (2007) 11185-11191.

[17] D.Miyoshi, K.Nakamura, H.Tateishi-Karimata, T.Ohmichi, N.Sugimoto. Hydration of WatsonCrick Base Pairs and Dehydration of Hoogsteen Base Pairs Inducing Structural Polymorphism under Molecular Crowding Conditions, J. Am. Chem. Soc. 131 (2009) 3522-3531.

[18] D.Miyoshi, H.Karimata, N.Sugimoto. Hydration regulates thermodynamics of G-quadruplex formation under molecular crowding conditions, J. Am. Chem. Soc. 128 (2006) 7957-7963.

[19] M.Vorlickova, K.Bednarova, J.Kypr. Ethanol is a better inducer of DNA guanine tetraplexes than potassium cations, Biopolymers 82 (2006) 253-260.

[20] B.Heddi, A.T.Phan. Structure of human telomeric DNA in crowded solution, J. Am. Chem. Soc. 133 (2011) 9824-9833.

[21] M.C.Miller, R.Buscaglia, J.B.Chaires, A.N.Lane, J.O.Trent. Hydration Is a Major Determinant of the G-Quadruplex Stability and Conformation of the Human Telomere 3' Sequence of d(AG(3)(TTAG(3))(3)), J. Am. Chem. Soc. (2010). 
[22] F.Rosu, V.Gabelica, L.Joly, G.Gregoire, E.De Pauw. Zwitterionic i-motif structures are preserved in DNA negatively charged ions produced by electrospray mass spectrometry, Phys. Chem. Chem. Phys. 12 (2010) 13448-13454.

[23] V.Gabelica, E.De Pauw, F.Rosu. Interaction between antitumor drugs and double-stranded DNA studied by electrospray ionization mass spectrometry, J. Mass Spectrom. 34 (1999) 1328-1337.

[24] K.B.Turner, S.A.Monti, D.Fabris. Like polarity ion/ion reactions enable the investigation of specific metal interactions in nucleic acids and their noncovalent assemblies, J. Am. Chem. Soc. 130 (2008) 13353-13363.

[25] S.E.Evans, M.A.Mendez, K.B.Turner, L.R.Keating, R.T.Grimes, S.Melchoir, V.A.Szalai. Endstacking of copper cationic porphyrins on parallel-stranded guanine quadruplexes, J. Biol. Inorg. Chem. 12 (2007) 1235-1249.

[26] W.M.David, J.Brodbelt, S.M.Kerwin, P.W.Thomas. Investigation of quadruplex oligonucleotidedrug interactions by electrospray ionization mass spectrometry, Anal. Chem. 74 (2002) 20292033.

[27] Y.Liu, B.Zheng, X.Xu, G.Yuan. Probing the binding affinity of small-molecule natural products to the G-quadruplex in C-myc oncogene by electrospray ionization mass spectrometry, Rapid Commun. Mass Spectrom. 24 (2010) 3072-3075.

[28] H.Li, G.Yuan, D.Du. Investigation of formation, recognition, stabilization, and conversion of dimeric G-quadruplexes of HIV-1 integrase inhibitors by electrospray ionization mass spectrometry, J. Am. Soc. Mass Spectrom. 19 (2008) 550-559.

[29] M.Vairamani, M.L.Gross. G-quadruplex formation of thrombin aptamer detected by electrospray ionization mass spectrometry, J. Am. Chem. Soc. 125 (2003) 42-43.

[30] S.E.Pierce, C.L.Sherman, J.Jayawickramarajah, C.M.Lawrence, J.L.Sessler, J.S.Brodbelt. ESI-MS characterization of a novel pyrrole-inosine nucleoside that interacts with guanine bases, Anal. Chim. Acta 627 (2008) 129-135.

[31] L.P.Bai, M.Hagihara, Z.H.Jiang, K.Nakatani. Ligand binding to tandem G quadruplexes from human telomeric DNA, ChemBioChem. 9 (2008) 2583-2587.

[32] B.Datta, M.E.Bier, S.Roy, B.Armitage. Quadruplex formation by a guanine-rich PNA oligomer, J. Am. Chem. Soc. 127 (2005) 4199-4207.

[33] J.Gidden, E.S.Baker, A.Ferzoco, M.T.Bowers. Structural motifs of DNA complexes in the gas phase, Int. J. Mass Spectrom. 240 (2004) 183-193. 
[34] Y.Krishnan-Ghosh, D.S.Liu, S.Balasubramanian. Formation of an interlocked quadruplex dimer by d(GGGT), J. Am. Chem. Soc. 126 (2004) 11009-11016.

[35] K.C.Gornall, S.Samosorn, B.Tanwirat, A.Suksamrarn, J.B.Bremner, M.J.Kelso, J.L.Beck. A mass spectrometric investigation of novel quadruplex DNA-selective berberine derivatives, Chem. Commun. 46 (2010) 6602-6604.

[36] W.Li, M.Zhang, J.L.Zhang, H.Q.Li, X.C.Zhang, Q.Sun, C.M.Qiu. Interactions of daidzin with intramolecular G-quadruplex, FEBS Lett. 580 (2006) 4905-4910.

[37] K.C.Porter, J.L.Beck. Assessment of the gas phase stability of quadruplex DNA using travelling wave ion mobility mass spectrometry, Int. J. Mass Spectrom. 304 (2011) 195-203.

[38] V.Gabelica. Determination of equilibrium association constants of ligand-DNA complexes by electrospray mass spectrometry, Methods Mol. Biol. 613 (2010) 89-101.

[39] F.Rosu, V.Gabelica, H.Poncelet, E.De Pauw. Tetramolecular G-quadruplex formation pathways studied by electrospray mass spectrometry, Nucleic Acids Res. 38 (2010) 5217-5225.

[40] N.B.Cech, C.G.Enke. Practical implications of some recent studies in electrospray ionization fundamentals, Mass Spectrom. Rev. 20 (2001) 362-387.

[41] V.Gabelica, N.Galic, F.Rosu, C.Houssier, E.De Pauw. Influence of response factors on determining equilibrium association constants of non-covalent complexes by electrospray ionization mass spectrometry, J. Mass Spectrom. 38 (2003) 491-501.

[42] M.C.Kuprowski, L.Konermann. Signal response of coexisting protein conformers in electrospray mass spectrometry, Anal. Chem. 79 (2007) 2499-2506.

[43] S.Mathur, M.Badertscher, M.Scott, R.Zenobi. Critical evaluation of mass spectrometric measurement of dissociation constants: accuracy and cross-validation against surface plasmon resonance and circular dichroism for the calmodulin-melittin system, Phys. Chem. Chem. Phys. 9 (2007) 6187-6198.

[44] J.M.Wilcox, D.L.Rempel, M.L.Gross. Method of measuring oligonucleotide-metal affinities: Interactions of the thrombin binding aptamer with $\mathrm{K}^{+}$and $\mathrm{Sr}^{2+}$, Anal. Chem. 80 (2008) 23652371.

[45] M.C.Jecklin, D.Touboul, C.Bovet, A.Wortmann, R.Zenobi. Which electrospray-based ionization method best reflects protein-ligand interactions found in solution? a comparison of ESI, nanoESI, and ESSI for the determination of dissociation constants with mass spectrometry, J. Am. Soc. Mass Spectrom. 19 (2008) 332-343. 
[46] V.Gabelica, F.Rosu, E.De Pauw. A Simple Method to Determine Electrospray Response Factors of Noncovalent Complexes, Anal. Chem. 81 (2009) 6708-6715.

[47] S.Masiero, R.Trotta, S.Pieraccini, T.S.De, R.Perone, A.Randazzo, G.P.Spada. A non-empirical chromophoric interpretation of CD spectra of DNA G-quadruplex structures, Org. Biomol. Chem. 8 (2010) 2683-2692.

[48] K.Vékey. Internal energy effects in mass spectrometry, J. Mass Spectrom. 31 (1996) 445-463.

[49] S.A.McLuckey. Principles of collisional activation in analytical mass spectrometry, J. Am. Soc. Mass Spectrom. 3 (1991) 599-614.

[50] V.Gabelica, E.De Pauw. Internal energy and fragmentation of ions produced in electrospray sources, Mass Spectrom. Rev. 24 (2005) 566-587. 